\title{
How can the R.E.N.A.L. nephrometry scoring system aid management of a solid renal mass?
}

\author{
MH Wong *, KY Cho, KL Ho, KW Wong, CT Lai, CM Man, MK Yiu
}

\section{A B S T R A C T}

Objectives: To investigate use of the R.E.N.A.L. nephrometry score in relation to the choice of treatment and postoperative complications for renal masses.

Design: Case series.

Setting: A tertiary referral hospital in Hong Kong.

Patients: Data of patients undergoing nephrectomy were collected retrospectively from a clinical database and analysed. A R.E.N.A.L. nephrometry score was allocated to each renal tumour by a blinded qualified radiologist, utilising computerised imaging systems. Patient demographics, choice of surgery (radical vs partial), and approaches (open vs minimally invasive) were analysed with respect to their R.E.N.A.L. score.

Results: In all, 74 patients were included during the study period, of which 38 underwent partial nephrectomyand 36 underwent radical nephrectomy. No differences between the groups were found with respect to patient demographics. There were significant differences between the partial and radical nephrectomy groups in terms of their mean nephrometry score (6.9 vs $9.3, \mathrm{P}<0.001)$. The mean nephrometry sum was also significantly different in the open approach versus the minimally invasive approach in patients having partial nephrectomy ( 7.8 vs $6.0, \mathrm{P}=0.001$ ). There was no difference in the postoperative 90-day morbidity and mortality in the partial nephrectomy and radical nephrectomy groups.

Conclusions: The R.E.N.A.L. nephrometry score of a renal mass correlated significantly with our choice of surgery (partial vs radical) and our approach to surgery (open vs minimally invasive surgery), particularly in the partial nephrectomy group. It does not, however, correlate with postoperative complications. The nephrometry score provides a useful tool for objectively describing renal mass characteristics and enhancing better communication for the operative planning directed at renal masses.

\section{Hong Kong Med J 2014;20:37-44}

DOI: $10.12809 / \mathrm{hkmj} 133920$

\author{
${ }^{1}$ MH Wong *, FHKAM (Surgery) \\ ${ }^{2}$ KY Cho, FRCR \\ ${ }^{1}$ KL Ho, FHKAM (Surgery) \\ ${ }^{1} \mathrm{KW}$ Wong, MRCS (Surgery) \\ ${ }^{1}$ CT Lai, MRCS (Surgery) \\ ${ }^{1}$ CM Man, MRCS (Surgery) \\ ${ }^{1}$ MK Yiu, FHKAM (Surgery)
}

1 Division of Urology, Department of Surgery
2 Department of Radiology
The University of Hong Kong, Queen Mary Hospital, Pokfulam, Hong Kong

* Corresponding author: edwong56@gmail.com

New knowledge added by this study

- We externally validated the use of the R.E.N.A.L. nephrometry scoring system to differentiate choice of surgery (radical vs partial nephrectomy) and approach (open vs minimally invasive), which was not shown in previous studies.

- We are able to qualify the weighting of individual parameters of the R.E.N.A.L. nephrometry scoring system on decision-making.

Implications for clinical practice or policy

- Application of R.E.N.A.L. nephrometry scoring preoperatively may be used as a guide to the complexity and choice of surgery in patients with small solid renal masses. It also serves as a tool for patient counselling, with reference to postoperative outcomes.

- Widespread use of this score may act as communication tools among specialists, such that direct comparisons of data and study results can be achieved.

\section{Introduction}

The annual incidence of renal cell carcinoma (RCC) in Hong Kong has increased steadily over the past 10 years reaching a rate of 5.9 cases per 100000 inhabitants. ${ }^{1}$ Surgical management remains the main treatment modality. With advances and ready availability of imaging, including screening by ultrasonography, more RCCs are diagnosed at an early stage (ie T1). The treatment modalities of these localised renal masses include radical nephrectomy or partial nephrectomy, in the form of an open or laparoscopic (with or without robotic-assisted) 


\section{R.E.N.A.L. 評分如何協助治理腎臟腫瘤？}

\section{王明晧、曹君彥、何崑崙、黃家榮、賴俊廷、文芷薇、姚銘廣}

目的：探討R.E.N.A.L.評分與腎藏腫瘤的治療方法和術後併發症的關 係。

設計：病例系列。

安排：香港一所提供第三層轉介醫療服務的醫院。

患者：從臨床數據庫取得曾進行腎切除的患者資料並進行回顧分析。 由被設盲的放射科專科醫生利用電腦成像系統為每個病例的腎腫瘤 進行R.E.N.A.L.評分, 並分析患者的人口學資料、所選擇的手術方法 (根治或局部切除) 及模式 (開放式與微創) 與R.E.N.A.L.得分的關 係。

結果：共分析了 74名患者, 其中38例接受腎部份切除術, 另36例接 受根治性腎切除術。兩組患者的人口學資料並無差異。根據患者的 R.E.N.A.L.平均得分, 進行局部和根治性腎切除術的患者有顯著差異 （6.9 比 $9.3, \mathrm{P}<0.001)$ 。在局部切除術的組別當中, R.E.N.A.L.平 均得分在開放式手術與微創手術明顯不同（7.8比6.0，P=0.001）。 術後 90 天的發病率和死亡率在腎部分切除術和根治性腎切除術組別之 間並無差異。

結論：腎臟腫瘤的R.E.N.A.L.得分與選擇的手術方法（根治或局部切 除）以及手術模式（開放式與微創）顯著相關, 尤其是在腎部分切除 術組別中, 但R.E.N.A.L.得分與術後併發症無關。R.E.N.A.L.評分能客 觀描繪腎腫瘤的特徵, 並有助手術規劃。 approach, as well as other form of ablative therapy. Several large, retrospective studies and the recently published European Organization for Research and Treatment of Cancer randomised trial ${ }^{2}$ have confirmed that the oncological outcomes of partial nephrectomy and radical nephrectomy are equivalent. The advantages of radical nephrectomy include better preservation of renal function and prevention of renal failure, lower cardiovascular morbidity, and better overall survival. ${ }^{3}$ Although nephronsparing surgery has slightly higher complication rate compared with radical nephrectomy, ${ }^{4}$ most international guidelines recommend the former as the standard treatment for solitary renal tumours up to a diameter of $7 \mathrm{~cm}$, whenever technically feasible., ${ }^{5,6}$ In the US population, utilisation of such techniques has recently been reported to be low, partly due to lack of technical advancements and publicity about possible adverse long-term consequences. ${ }^{7}$

Decisions on the choice of surgery mostly depend on the size and location of the tumour. Other external factors, such as the surgeon's training, practice pattern, operating centre facilities, and hardware available, have a major impact on the choice of approaches and operation to be performed. In the presence of multiple treatment options, an objective way to describe the complexity of renal masses and to accurately assess the risks of postoperative complications is important for patient counselling and clinical decision-making. Scoring systems have therefore been developed and validated, and to date three are available for clinical use. . $^{8-10}$

Herein, we report our investigation into using the R.E.N.A.L. nephrometry score, as developed by Kutikov and Uzzo in 2009, ${ }^{8}$ and its relationship to the choice of treatment and postoperative complications.

\section{Methods}

Data about patients having renal tumours treated by total nephrectomy in Queen Mary Hospital during the period of January 2006 to December 2011 were retrieved retrospectively from a clinical database and analysed. Patients who had not had preoperative computed tomography and threedimensional reconstruction (available in the Queen Mary Hospital radiological department) were excluded, so as to standardise the radiographic characteristic of the renal tumours under study. This involved allocating a R.E.N.A.L. nephrometry score to each renal tumour utilising computerised imaging systems (GE Advantage Workstations; General Electric Healthcare, US) by a blinded qualified radiologist. The R.E.N.A.L. score was described in 2009 and includes the assessment of tumour (R)adius (size at the maximal diameter), (E)xophytic/ endophytic properties, $(\mathrm{N})$ earness of tumour to the collecting system or sinus, (A)nterior/posterior descriptor, and (L)ocation relative to polar lines. Standardised points (1-3 points per descriptor) were assigned onto each parameter, except the anterior or posterior component as originally described by Kutikov and $\mathrm{Uzzo}^{8}$ (Table 1). Radius was measured as the maximum diameter of the tumour in centimetres and points were allocated as $1(\leq 4 \mathrm{~cm}), 2(>4$ but $<7$ $\mathrm{cm})$, and $3(\geq 7 \mathrm{~cm})$. Exophytic/endophytic points assigned were 1 when $50 \%$ or more of the tumour was exophytic, 2 when less than $50 \%$ was exophytic, and 3 when it was entirely endophytic. For nonspherical or asymmetrically located tumours, the predominant feature on any axis (not just the axial or coronal axis) was considered with reference to the renal cortex. The $\mathrm{N}$ component was measured as the distance of the deepest portion of the tumour to the collecting system and points were allocated as $1(\geq 7$ $\mathrm{mm}$ ), 2 ( $>4$ but $<7 \mathrm{~mm}$ ), and 3 (invading, touching or within $4 \mathrm{~mm}$ ). Anterior/posterior location of the tumour was designated as a non-numerical suffix that describes the location of the tumour with respect to the kidney midline plane as assessed on axial images. When the mass was located at the tip of the renal poles or lay on the coronal plane where a meaningful anterior or posterior designation was not possible, the suffix " $x$ " was assigned. The location score was assigned as the position of the mass relative to polar lines. The polar line was assigned as the plane of 
the kidney above or below which the medial lip of demographics, including age, gender, preoperative parenchyma was interrupted by the renal sinus fat, renal function, and estimated glomerular filtration vessels or the collecting system and best located in rate (eGFR) as calculated by Chronic Kidney Disease the coronal plane. Two polar lines were measured Epidemiology Collaboration (CKD-EPI) equations for each renal unit. The position of the renal tumour were logged. ${ }^{11}$ In addition, the American Society with respect to the polar lines was measured and a of Anesthesiologists (ASA) class, ${ }^{12}$ chronic kidney score allocated as described in Table 1 . Nephrometry disease stage, mode of surgery (radical vs partial), classes in terms of complexity were allocated as low approaches (open vs minimally invasive surgery (4-6), moderate (7-9), and high (10-12) based on the [MIS]), and ischaemic time were analysed with sum of scores allocated to each parameter. Patient respect to their R.E.N.A.L. score and classes. The

TABLE I. R.E.N.A.L. nephrometry scoring system ${ }^{8}$

\begin{tabular}{|c|c|c|c|}
\hline & \multicolumn{3}{|c|}{ R.E.N.A.L. score } \\
\hline & 1 Point & 2 Points & 3 Points \\
\hline (R)adius (maximal diameter in $\mathrm{cm}$ ) & $\leq 4$ & $>4$ but $<7$ & $\geq 7$ \\
\hline (E)xophytic/endophytic properties & $\geq 50 \%$ & $<50 \%$ & Entirely endophytic \\
\hline $\begin{array}{l}\text { (N)earness of tumour to the collecting } \\
\text { system or sinus ( } \mathrm{mm})\end{array}$ & $\geq 7$ & $>4$ but $<7$ & $\leq 4$ \\
\hline (A)nterior/posterior & \multicolumn{3}{|c|}{ No points given. Mass assigned a descriptor of $a, p$, or $x$} \\
\hline (L)ocation relative to polar lines & $\begin{array}{l}\text { Entirely above the upper or } \\
\text { below the lower polar line }\end{array}$ & Lesion crosses polar line & $\begin{array}{l}>50 \% \text { Of the mass across polar line, or } \\
\text { mass crosses the axial renal midline, or } \\
\text { mass is entirely between the polar lines }\end{array}$ \\
\hline
\end{tabular}

TABLE 2. Basic demographics of partial nephrectomy and radical nephrectomy groups

\begin{tabular}{|c|c|c|c|}
\hline \multirow[t]{2}{*}{ Demographics } & \multicolumn{2}{|c|}{ No. of patients or mean \pm standard deviation } & \multirow[t]{2}{*}{$P$ value } \\
\hline & Partial nephrectomy $(n=38)$ & Radical nephrectomy $(n=36)$ & \\
\hline Gender & & & 0.980 \\
\hline Male & 21 & 20 & \\
\hline Female & 17 & 16 & \\
\hline Age (years) & $58 \pm 15$ & $62 \pm 14$ & 0.207 \\
\hline Mean preoperative creatinine (mmol/L) & $97 \pm 64$ & $158 \pm 228$ & 0.118 \\
\hline Mean preoperative eGFR (mL/min) & $77 \pm 20$ & $65 \pm 27$ & 0.039 \\
\hline CKD stage & & & 0.292 \\
\hline 1 & 11 & 9 & \\
\hline 2 & 12 & 16 & \\
\hline 3 & 4 & 6 & \\
\hline 5 & 0 & 3 & \\
\hline N/A & 11 & 2 & \\
\hline ASA class & & & 0.300 \\
\hline 1 & 3 & 2 & \\
\hline 2 & 28 & 21 & \\
\hline 3 & 6 & 9 & \\
\hline $\mathrm{N} / \mathrm{A}$ & 1 & 4 & \\
\hline Pathology & & & 0.072 \\
\hline RCC (clear cell) & 24 & 28 & \\
\hline RCC (chromophobe) & 1 & 2 & \\
\hline Oncocytoma & 1 & 3 & \\
\hline AML & 9 & 1 & \\
\hline Others & 3 & 2 & \\
\hline
\end{tabular}

Abbreviations: eGFR = estimated glomerular filtration rate; $\mathrm{CKD}=$ chronic kidney disease; $\mathrm{N} / \mathrm{A}$ = not available; $\mathrm{ASA}=$ American

Society of Anesthesiologists; $\mathrm{RCC}=$ renal cell carcinoma; $\mathrm{AML}=$ angiomyolipoma 
90-day postoperative morbidity and mortality were retrieved according to the Clavien-Dindo system. ${ }^{13}$ Continuous variables were analysed with Student's $t$ test and categorical variables by the Chi squared and Fisher's exact tests. Any P value of $<0.05$ was taken as statistically significant. All data were analysed with the Statistical Package for the Social Sciences (Windows version 18.0; SPSS Inc, Chicago [IL], US).

\section{Results}

There were 74 patients included during this study period, of which 38 underwent partial nephrectomy (group 1) and 36 underwent radical nephrectomy (group 2). There were 41 males and 33 females. No statistical differences were found between the groups in terms of gender distribution, age, preoperative creatinine level, ASA class, or chronic kidney disease stage, although the mean eGFR was significantly lower in the radical nephrectomy group (65 vs $77 \mathrm{~mL} / \mathrm{min}, \mathrm{P}=0.039$; Table 2). The final pathology of the majority of our patients was clear-cell RCC $(n=52)$, and the remainder suffered from angiomyolipoma $(n=10)$, oncocytoma $(n=4)$, chromophobe RCC $(n=3)$, and others $(n=5)$. There were significant differences between the partial and radical nephrectomy groups in terms of their mean nephrometry score (6.9 vs $9.3, \mathrm{P}<0.001)$. Individual parameters of the R.E.N.A.L. score in terms of radius $(\mathrm{P}<0.001)$, nearest to the collecting system $(\mathrm{P}<0.001)$, and locations relative to polar lines $(\mathrm{P}=0.017)$ were significantly different in the two groups, but there was no significant difference in terms of exophytic components or anterior/posterior location (Table 3).

Further analysis of the partial nephrectomy patients revealed that respective mean nephrometry scores of open versus MIS were 7.8 vs $6.0(\mathrm{P}=0.001)$, and in particular the nearest components were significantly different $(\mathrm{P}<0.001$; Table 4$)$. Such a difference was evident for the radical nephrectomy group. The overall 90-day morbidity in our study cohort was low, and included urinary leakage $(n=1)$ and bleeding warranting embolisation $(n=1)$ in the partial nephrectomygroup, and intestinal obstruction $(n=1)$ in the radical nephrectomy group. None of our patients received a postoperative transfusion. Mortality at 90 days in the radical nephrectomy group $(n=1)$ was in a patient with metastatic RCC undergoing cytoreductive nephrectomy. There was no difference in postoperative 90-day morbidity and mortality between the two groups, even after stratification according to mean nephrometry score or with respect to different classes (Table 5). Ischaemic time was significantly higher for patients in higher nephrometry classes in the partial nephrectomy group (36 mins vs 51 mins vs 80

TABLE 3. Association between choice of surgery with nephrometry score and individual parameters

\begin{tabular}{|c|c|c|c|}
\hline \multirow[t]{2}{*}{ Variable } & \multicolumn{2}{|c|}{ Mean \pm standard deviation or No. of patients } & \multirow[t]{2}{*}{$P$ value } \\
\hline & Partial nephrectomy $(n=38)$ & Radical nephrectomy $(n=36)$ & \\
\hline Nephrometry sum & $6.9 \pm 1.7$ & $9.3 \pm 1.5$ & $<0.001$ \\
\hline (R)adius & & & $<0.001$ \\
\hline 1 & 33 & 5 & \\
\hline 2 & 4 & 14 & \\
\hline 3 & 1 & 17 & \\
\hline (E)xophytic/endophytic properties & & & 0.858 \\
\hline 1 & 16 & 16 & \\
\hline 2 & 19 & 19 & \\
\hline 3 & 3 & 1 & \\
\hline (N)earness of tumour to the collecting system or sinus & & & $<0.001$ \\
\hline 1 & 11 & 0 & \\
\hline 2 & 9 & 1 & \\
\hline 3 & 18 & 35 & \\
\hline (A)nterior or posterior or $\mathrm{x}$ & & & 0.515 \\
\hline a & 12 & 14 & \\
\hline $\mathrm{p}$ & 13 & 8 & \\
\hline$x$ & 13 & 14 & \\
\hline (L)ocation relative to polar lines & & & 0.017 \\
\hline 1 & 16 & 9 & \\
\hline 2 & 9 & 3 & \\
\hline 3 & 13 & 24 & \\
\hline
\end{tabular}


mins, $\mathrm{P}=0.008$; Table 6); all three patients with high nephrometry scores underwent open surgery using cold ischaemia with ice sludge surface cooling, thus explaining the difference in ischaemic time.

\section{Discussion}

The standard care of patients with a solid renal mass is excision. Partial nephrectomy has become

TABLE 4. Comparisons of surgical approaches in partial nephrectomy in relation to nephrometry score

\begin{tabular}{|c|c|c|c|}
\hline \multirow[t]{2}{*}{ Variable } & \multicolumn{2}{|c|}{ Mean \pm standard deviation or No. of patients } & \multirow[t]{2}{*}{$P$ value } \\
\hline & Open surgery $(n=19)$ & Minimally invasive surgery $(n=19)$ & \\
\hline Nephrometry sum & $7.8 \pm 1.5$ & $6.0 \pm 1.5$ & 0.001 \\
\hline (R)adius & & & 0.597 \\
\hline 1 & 16 & 17 & \\
\hline 2 & 2 & 2 & \\
\hline 3 & 1 & 0 & \\
\hline (E)xophytic/endophytic properties & & & 0.132 \\
\hline 1 & 5 & 11 & \\
\hline 2 & 13 & 7 & \\
\hline 3 & 1 & 1 & \\
\hline (N)earness of tumour to the collecting system or sinus & & & $<0.001$ \\
\hline 1 & 2 & 9 & \\
\hline 2 & 2 & 7 & \\
\hline 3 & 15 & 3 & \\
\hline (A)nterior or posterior or $\mathrm{x}$ & & & 0.189 \\
\hline a & 4 & 8 & \\
\hline$p$ & 9 & 4 & \\
\hline$x$ & 6 & 7 & \\
\hline (L)ocation relative to polar lines & & & 0.406 \\
\hline 1 & 6 & 10 & \\
\hline 2 & 5 & 4 & \\
\hline 3 & 8 & 5 & \\
\hline
\end{tabular}

TABLE 5. Complications

\begin{tabular}{|c|c|c|c|}
\hline Complication & Partial nephrectomy & Radical nephrectomy & $P$ value \\
\hline None & 26 & 31 & \\
\hline Conversion & 3 & 0 & \\
\hline Bleeding require embolisation & 1 & 0 & \\
\hline Urinary leakage & 1 & 0 & \\
\hline Intestinal obstruction & 0 & 1 & \\
\hline $\begin{array}{l}\text { Others (ileus, AF, atelectasis, UTI, retention, } \\
\text { gout, wound gapping) }\end{array}$ & 7 & 4 & \\
\hline Total & 38 & 36 & 0.243 \\
\hline \multicolumn{4}{|l|}{ Complication: Clavien grade } \\
\hline 0 & 28 & 30 & \\
\hline 1 & 8 & 4 & \\
\hline 2 & 0 & 0 & \\
\hline 3 & 2 & 1 & \\
\hline 4 & 0 & 0 & \\
\hline 5 & 0 & 1 & \\
\hline Total & 38 & 36 & 0.153 \\
\hline
\end{tabular}

Abbreviations: $\mathrm{AF}=$ atrial fibrillation; $\mathrm{UTI}=$ urinary tract infection 
TABLE 6. Operative parameters of partial nephrectomy stratified by nephrometry class

\begin{tabular}{lrccc}
\hline Parameter & \multicolumn{3}{c}{ Nephrometry class } & P value \\
\cline { 2 - 4 } & Low (4-6) & Moderate (7-9) & High (10-12) & \\
\hline Operating time (mins) & 332 & 338 & 329 & 0.196 \\
Blood loss (mL) & 327 & 493 & 169 & 0.155 \\
Intra-operative transfusion & 0 & 1 & 0 & 0.630 \\
Ischaemic time (mins) & 36 & 51 & 80 & 0.008 \\
\hline
\end{tabular}

the standard for T1a RCCs and more recent data support its use in larger tumours of up to $7 \mathrm{~cm}$ (ie T1b). Most internationally recognised guidelines support recourse to partial nephrectomy for T1a tumours whenever technically feasible, ${ }^{5,6}$ as data suggest comparable oncological outcomes with more favourable outcomes in terms of risk of renal failure warranting dialysis, cardiovascular morbidity, and even mortality. Approaches to the management of a solid renal mass include consideration of whether to remove the whole kidney or resect the tumour only and achieve a margin clear of pathology. Secondary consideration is given to the approach of the surgery, be it a traditional open one or MIS (purely laparoscopic or robotic-assisted laparoscopic). Although the latter is technically more demanding and has more postoperative complications (blood loss, recourse to transfusions, and urinary leakage), many high-volume centres show favourable results in experienced hand. ${ }^{14}$

Many factors contribute to the choice of surgery and mode of approach. They include hospital infrastructures and patient volume, experience and training history of the relevant surgeons, patient preference, and most importantly tumour characteristics. Traditionally, clinical decisions were based mostly on the first of these factors, resulting in heterogeneous clinical choices and operative results. Even when only tumour characteristics were taken into account, there was wide heterogeneity in definitions, such as centrality or hilar location, and makes direct comparison of results between studies difficult and impractical.

The concept of nephrometry was proposed as a tool to objectively assess the complexity of a solid renal mass. To date there are three studies of largely nephrometric systems. They are the R.E.N.A.L nephrometry score proposed by Kutikov and Uzzo in $2009,{ }^{8}$ the preoperative aspects and dimensions used for an anatomical (PADUA) classification of renal tumours by Ficarra et al in 2009, ${ }^{9}$ and the C-index method proposed by Simmons et al in 2010. ${ }^{10}$ Most studies utilise the nephrometry scales in patients undergoing partial nephrectomy. The three methods made use of different parameters to assess the locations of the tumour in relation to various important structures of the kidney, and to predict the technical difficulty that might be encountered during nephron-sparing surgery of the target lesion. They have been reviewed as new tools that can guide surgical decision-making to improve academic reporting, risk assessment of complications, and prediction of functional outcomes.

The R.E.N.A.L. nephrometry score is one of the most studied scoring systems with numerous articles describing its use in clinical practice. The original description of the score was to set a standard reporting system, and its use suggested a relationship between renal mass anatomy, pathology, and prognosis. ${ }^{8}$ Assessments of inter-observer variability confirm their reproducibility and interobserver agreement was robust across specialties and levels of training. ${ }^{15-18}$ Later studies showed that high R.E.N.A.L. scores were associated with higher major complication rates than those with intermediate or low scores. ${ }^{15,19}$ Moreover, multivariate analysis revealed that prolonged operating time and highcomplexity nephrometry score category were independent predictors of major complications. ${ }^{19}$ Other reports demonstrated that the R.E.N.A.L. score correlated with both tumour grade $(P<0.0001)$ and histology $(\mathrm{P}<0.0001)$, such that as tumour size increases there would be a greater probability of malignancy, including high-grade and clear-cell tumour on histology. ${ }^{20,21}$ Nomograms have been developed based on study results to preoperatively predict the likelihood of malignant and high-grade pathology of an enhancing renal mass,$^{20}$ and such systems have been externally validated. ${ }^{22}$ Other studies have demonstrated the association of nephrometry scores with use of ischaemia in partial nephrectomy, ${ }^{15}$ warm ischaemia time, ${ }^{23}$ choice of surgery (partial vs radical nephrectomy), ${ }^{17,24,25}$ need of conversion to radical nephrectomy, ${ }^{23}$ changes in the percent functional volume preserved and perioperative functional decrease, ${ }^{26}$ long-term renal functional outcome following partial nephrectomy, ${ }^{27}$ and postoperative urinary leakage. ${ }^{28}$ In particular R.E.N.A.L. scores were higher in patients with partial nephrectomy who developed complications than in partial nephrectomy patients who did not (6.9 vs 6.0, $\mathrm{P}=0.02$ ). No corresponding differences were found in patients having radical nephrectomy $(\mathrm{P}=0.99) .{ }^{29}$ Other studies investigating their applications on robotic partial nephrectomy have shown incongruent results. In one study, Mufarrij et a ${ }^{30}$ did not show the ability of this scoring system to predict perioperative outcomes in robotic-assisted partial nephrectomy. Others found significant correlations of the score with increased warm ischaemia time, blood loss, complications, and length of hospital stay ${ }^{31,32}$ in patients undergoing robotic and laparoscopic partial nephrectomy. Clinical application of such anatomical classification systems has gained popularity in selecting cases suitable for alternative treatment of 
small renal masses (such as by thermal ablation). ${ }^{33}$ Available data so far show more evidence to support the use of this scoring system to make treatment decision more objective for renal masses. ${ }^{34,35}$

The results of our study clearly demonstrate a positive correlation of R.E.N.A.L. scores with the choice of nephrectomy (partial vs radical), in terms of the total summed scores and individual parameters including radius (size), location nearest to the collecting system, and relationship to polar lines. These findings support the idea that clinical decisions based solely on the size of tumours are oversimplified and other anatomical factors should enter overall considerations. We did not find significant correlations for other individual parameters, such as exophytic components and anterior/posterior location. This was in contrast to a previous study which espoused the relevance of such components to the choice of ablative therapy (radiofrequency, cryoablation, or partial nephrectomy) as originally described by Kutikov and Uzzo. ${ }^{8}$ Another significant finding was the correlation between the score and the choice of approach in partial nephrectomy. It was shown that with an increase in mean nephrometry score or class, there was a trend towards choosing open rather than a MIS approach. This signifies that whenever partial nephrectomy is feasible, the open method is preferred for more complex tumours and that this practice can be based on an objective scoring system. However, this was not observed in our radical nephrectomy group, which echoed a previous study finding and like the original description aimed at partial nephrectomy (not radical nephrectomy). The significant correlation of R.E.N.A.L. class with ischaemic time may be useful to guide the choice of open approaches for partial nephrectomy in the presence of a renal tumour with a high score. This could facilitate the safe use of cold ischaemia so as to maximise preservation of renal function.

Our results were contrary to previous investigators reporting that the R.E.N.A.L. score was not associated with presence or severity of complications in both patient groups in terms of their mean score or class. This could be explained by the relatively low frequency of major complications in our study cohort (5.4\%) and in the small sample size. With more prospective data available, we believe similar correlations of the score with the frequency of postoperative complications and perioperative outcomes would be revealed.

An inherent limitation of our study was that it was retrospective with respect to data collection and analysis. A second limitation was the exclusion of many patients due to unavailability of satisfactory quality images for the calculation of scores to make direct comparisons. A third limitation of the R.E.N.A.L. score per se was that the weight given to individual components contributed to the total score; numerical values were allocated arbitrarily and still await validation. Although ours is one of the few studies that demonstrate the association of this score and individual parameters on the choice of surgery rather than sole reliance on tumour size, we still have to define a single value in this scoring system below which we can confidently recommend partial nephrectomy. Moreover, other confounding factors such as the surgeon's experience and learning curve data were not available for analysis, and may heavily influence clinical decisions.

Future directions of studies and clinical utilisation of such a scoring system will aim to define different weightings for individual components contributing to the total score. Other studies may aim at enhancing the reproducibility and predictability of such tools, so that direct comparison can be made with other centres. Are we doing better than eyeballing when managing a solid renal mass? Maybe we are, but the use of the nephrometry score will enhance communication, documentation, and education for the coming younger generation of urologists. Lately, Simmons et $\mathrm{al}^{36}$ have described the integration of the R.E.N.A.L. and C-index scoring systems as diameter-axial-polar nephrometry (DAP). Initial results demonstrate the DAP scoring system to be simpler, to decrease measurement error, to improve performance characteristic, to make interpretation easier, and to exhibit a clear association with volume loss and late function after partial nephrectomy. More mature data will allow us to choose the best tools for our patients.

\section{Conclusions}

The R.E.N.A.L. nephrometry score of a solid renal mass shows a significant association with our choice of surgery (partial vs radical) and our approach to surgery (open vs MIS), particularly in patients receiving partial nephrectomy. Its association with postoperative complications was not demonstrated in this study. The score provides a useful tool to define the character of a renal mass objectively, aid clinical decision-making, and enhance communication between professionals with respect to the management of solid renal masses.

\section{References}

1. Hospital Authority. Hong Kong Cancer Registry. Available from: http://www3.ha.org.hk/cancereg/statistics.html. Accessed Jun 2013.

2. Van Poppel H, Da Pozzo L, Albrecht W, et al. A prospective randomized EORTC intergroup phase 3 study comparing the oncologic outcome of elective nephron-sparing surgery and radical nephrectomy for low-stage renal cell carcinoma. Eur Urol 2011;59:543-52.

3. Go AS, Chertow GM, Fan D, McCulloch CE, Hsu CY. Chronic kidney disease and the risks of death, cardiovascular events, and hospitalization. N Engl J Med 2004;351:1296- 
305

4. Van Poppel H, Da Pozzo L, Albrecht W, et al. A prospective randomized EORTC intergroup phase 3 study comparing the complications of elective nephron-sparing surgery and radical nephrectomy for low-stage renal cell carcinoma. Eur Urol 2007;51:1606-15.

5. Ljungberg B, Cowan NC, Hanbury DC, et al. EAU guidelines on renal cell carcinoma: the 2010 update. Eur Urol 2010;58:398-406.

6. Campbell SC, Novick AC, Belldegrun A, et al. Guideline for management of the clinical $\mathrm{T} 1$ renal mass. J Urol 2009;182:1271-9.

7. SmaldoneMC,KutikovA,Egleston B, etal.Assessingperformancetrendsinlaparoscopicnephrectomyandnephron-sparing surgery for localized renal tumors. Urology 2012;80:286-92.

8. Kutikov A, Uzzo RG. The R.E.N.A.L. nephrometry score: a comprehensive standardized system for quantitating renal tumor size, location and depth. J Urol 2009;182:844-53.

9. Ficarra V, Novara G, Secco S, et al. Preoperative aspects and dimensions used for an anatomical (PADUA) classification of renal tumors in patients who are candidates for nephronsparing surgery. Eur Urol 2009;56:786-93.

10. Simmons MN, Ching CB, Samplaski MK, Park CH, Gill IS. Kidney tumor location measurement using the $\mathrm{C}$ index method. J Urol 2010;183:1708-13.

11. Levey AS, Stevens LA, Schmid CH, et al. A new equation to estimate glomerular filtration rate. Ann Intern Med 2009;150:604-12.

12. American Society of Anesthesiologists. New classification of physical status. Anesthesiology 1963;24:111.

13. Dindo D, Demartines N, Clavien PA. Classification of surgical complications: a new proposal with evaluation in a cohort of 6336 patients and results of a survey. Ann Surg 2004;240:205-13.

14. Gill IS, Kavoussi LR, Lane BR, et al. Comparison of 1,800 laparoscopic and open partial nephrectomies for single renal tumors. J Urol 2007;178:41-6.

15. Hew MN, Baseskioglu B, Barwari K, et al. Critical appraisal of the PADUA classification and assessment of the R.E.N.A.L. nephrometry score in patients undergoing partial nephrectomy. J Urol 2011;186:42-6.

16. Kolla SB, Spiess PE, Sexton WJ. Interobserver reliability of the RENAL nephrometry scoring system. Urology 2011;78:592 4.

17. Weight CJ, Atwell TD, Fazzio RT, et al. A multidisciplinary evaluation of inter-reviewer agreement of the nephrometry score and the prediction of long-term outcomes. J Urol 2011;186:1223-8.

18. Montag S, Waingankar N, Sadek MA, Rais-Bahrami S, Kavoussi LR, Vira MA. Reproducibility and fidelity of the R.E.N.A.L. nephrometry score. J Endourol 2011;25:19258.

19. Simhan J, Smaldone MC, Tsai KJ, et al. Objective measures of renal mass anatomic complexity predicts rates of major complications following partial nephrectomy. Eur Urol 2011;60:724-30.

20. Kutikov A, Smaldone MC, Egleston BL, et al. Anatomic features of enhancing renal masses predicts malignant and high-grade pathology: a preoperative nomogram using the RENAL nephrometry score. Eur Urol 2011;60:241-8.

21. Satasivam P, Sengupta S, Rajarubendra N, Chia PH,
Munshey A, Bolton D. Renal lesions with low R.E.N.A.L nephrometry score are associated with more indolent renal cell carcinomas (RCCs) or benign histology: findings in an Australian cohort. BJU 2012;109 Suppl 3:44-7.

22. Wang HK, Zhu Y, Yao XD, et al. External validation of a nomogram using RENAL nephrometry score to predict high grade renal cell carcinoma. J Urol 2012;187:155560 .

23. Long JA, Arnoux V, Fiard G, et al. External validation of the RENAL nephrometry score in renal tumours treated by partial nephrectomy. BJU Int 2013;111:233-9.

24. Satasivam P, Rajarubendra N, Chia PH, Munshey A, Sengupta S, Bolton D. Trends in the use of nephron-sparing surgery (NSS) at an Australian tertiary referral centre: an analysis of surgical decision-making using the R.E.N.A.L. nephrometry scoring system. BJU Int 2012;109:1341-4.

25. Broughton GJ, Clark PE, Barocas DA, et al. Tumour size, tumour complexity, and surgical approach are associated with nephrectomy type in small renal cortical tumours treated electively. BJU Int 2012;109:1607-13.

26. Simmons MN, Hillyer SP, Lee BH, Fergany AF, Kaouk J, Campbell SC. Nephrometry score is associated with volume loss and functional recovery after partial nephrectomy. J Urol 2012;188:39-44.

27. Cha EK, Ng CK, Jeun B, et al. Preoperative radiographic parameters predict long-term renal impairment following partial nephrectomy. World J Urol 2013;31:817-22.

28. Bruner B, Breau RH, Lohse CM, Leibovich BC, Blute ML. Renal nephrometry score is associated with urine leak after partial nephrectomy. BJU Int 2011;108:67-72.

29. Rosevear HM, Gellhaus PT, Lightfoot AJ, Kresowik TP, Joudi FN, Tracy CR. Utility of the RENAL nephrometry scoring system in the real world: predicting surgeon operative preference and complication risk. BJU Int 2012;109:700-5.

30. Mufarrij PW, Krane LS, Rajamahanty S, et al. Does nephrometry scoring of renal tumors predict outcomes in patients selected for robot-assisted partial nephrectomy? J Endourol 2011;25:1649-53.

31. White MA, Haber GP, Autorino R, et al. Outcomes of robotic partial nephrectomy for renal masses with nephrometry score of $\geq 7$. Urology 2011;77:809-13.

32. Hayn MH, Schwaab T, Underwood W, Underood W, Kim HL. RENAL nephrometry score predicts surgical outcomes of laparoscopic partial nephrectomy. BJU Int 2011;108:87681.

33. Reyes J, Canter D, Putnam S, et al. Thermal ablation of the small renal mass: case selection using the R.E.N.A.L-Nephrometry Score. Urol Oncol 2013;31:1292-7.

34. Canter D, Kutikov A, Manley B, et al. Utility of the R.E.N.A.L. nephrometry scoring system in objectifying treatment decision-making of the enhancing renal mass. Urology 2011;78:1089-94.

35. Tobert CM, Kahnoski RJ, Thompson DE, Anema JG, Kuntzman RS, Lane BR. RENAL nephrometry score predicts surgery type independent of individual surgeon's use of nephron-sparing surgery. Urology 2012;80:157-61.

36. Simmons MN, Hillyer SP, Lee BH, Fergany AF, Kaouk J, Campbell SC. Diameter-axial-polar nephrometry: integration and optimization of R.E.N.A.L. and centrality index scoring systems. J Urol 2012;188:384-90. 